\title{
Hall Effects on MHD Flow of Second Grade Fluid through Porous Medium with Oscillatory Suction
}

\author{
B.Lakshmanna $^{1^{*}}$ and S.Venkateswarlu ${ }^{2}$ \\ ${ }^{1}$ Research Scholar, Dept of Mathematics, Rayalaseema University, Kurnool, Andhra Pradesh 518007, India. \\ ${ }^{2}$ Professor, Dept. of Mathematics, RGM College of Engineering and Technology, Nandyal, Kurnool, Andhra Pradesh, India.
}

\begin{abstract}
In this paper, we have considered Hall effects on the unsteady free convective two dimensional flow of a viscous incompressible electrically conducting second fluid over an infinite vertical porous plate under the influence of uniform transverse magnetic field with time dependent permeability, oscillatory suction. The governing equations of the flow field are solved by a regular perturbation method for small amplitude of the permeability. The closed form solutions for the velocity, temperature and concentration have been derived analytically and also its behavior is computationally discussed with reference to different flow parameters with the help of profiles. The skin fiction on the boundary, the heat flux in terms of the Nusselt number and rate of mass transfer in terms of Sherwood number are also obtained and their behavior computationally discussed.
\end{abstract}

Keywords: Heat transfer; mass transfer; oscillatory suction; porous medium; MHD flow; infinite vertical plates, Hall effects.

\section{INTRODUCTION}

Rates of many physiological functions, including the flow through blood vessels are affected by drugs. The rates of different biochemical reactions that are responsible for the contraction muscles, secretion of different materials. The rate at which the kidney cells perform the regulation of the volume of water/salts in the body is affected by drugs. The rate at which blood flows through arteries can also be enhanced/slowed down by the application of drugs. It may, however, be noted that the damaged structures/functions can only be repaired by drugs, but their restoration is not possible. This is the observation of the clinicians, when they treat patients suffering from various types of degenerative/ tissuedestroying diseases, including multiple atherosclerosis (narrowing of arterial lumen due to deposition of different fatty substances, cholesterol, etc.), arthritis, Alzheimer disease, Parkinson disease, heart failure. However, there are some drugs (e.g. antibiotics) that help the body in the damage repair process, when the damage takes place due to some infection. Several drugs (antacids, for example) produce effects, where the function of a cell remains unchanged and a receptor dose not have any cognition. Most of the antacids are bases that interact with stomach acid to neutralize it. Thus stomach acid is reduced simply through chemical reactions. Hall effects on MHD flow past over an accelerated plate in a rotating system in the presence of a magnetic field was examined by Deka [1]. Hydromagnetic channel flows in a rotating fluid system are investigated by researchers, Ghosh et al. [2]. Hydromagnetic convection flow in a rotating porous medium or in a channel partially filled by a porous medium with Hall effects are investigated by researchers such as Krishna et al. [3]. Chauhan and Agrawal [4-5], Dileep.D.C. [6] discussed the unsteady MHD flow of viscous incompressible and electrically conducting fluid through a porous medium adjacent to an accelerated impermeable plate in a rotating system taking Hall current into account, Heat transfer is also determined. Recently Veera Krishna and Prakash [7] discussed the unsteady flow of an incompressible viscous fluid in a rotating parallel plate channel bounded on one side by a porous bed under the influence of a uniform transverse magnetic field taking hall current into account. Ashaf et al. [8] discussed Solution of MHD flow past a vertical porous plate through a porous medium under oscillatory suction. B.Lakshmanna; S.Venkateswarlu[9] discussed Heat and Mass Transfer on MHD Flow of an incompressible fluid past an infinite vertical porous plate. Krishna and M.G. Reddy [10] discussed the unsteady MHD free convection in a boundary layer flow of an electrically conducting fluid through porous medium subject to uniform transverse magnetic field over a moving infinite vertical plate in the presence of heat source and chemical reaction. Krishna and G.S. Reddy [11] have investigated the simulation on the MHD forced convective flow through stumpy permeable porous medium (oil sands, sand) using Lattice Boltzmann method. Krishna and K.Jyothi [12] discussed the Hall effects on MHD Rotating flow of a visco-elastic fluid through a porous medium over an infinite oscillating porous plate with heat source and chemical reaction. B.S.K. Reddy et al.[13] investigated MHD flow of viscous incompressible nano-fluid through a saturating porous medium. Recently, Krishna et al. [14-17] discussed the MHD flows of an incompressible and electrically conducting fluid in planar channel. Veera Krishna et al. [18] discussed heat and mass transfer on unsteady MHD oscillatory flow of blood through porous arteriole. The effects of radiation and Hall current on an unsteady magnetohydrodynamic free convective flow in a vertical channel filled with a porous medium have been studied by Veera Krishna et al. [19].

In view of the above studies, in this paper, we have considered Hall effects on the unsteady free convective two dimensional flow of a viscous incompressible electrically conducting second grade fluid over an infinite vertical porous plate under 
the influence of uniform transverse magnetic field with time dependent permeability, oscillatory suction.

\section{FORMULATION AND SOLUTION OF THE PROBLEM:}

We considered the Hall effects on the unsteady MHD free convection two dimensional flow of an incompressible viscous electrically conducting second grade fluid with simultaneous heat and mass transfer over an infinite vertical plate through porous medium with time dependent permeability and oscillatory suction under the influence of uniform transverse magnetic field of strength $H_{0}$. The $z$-axis is taken along the plate and $x$-axis perpendicular to it and $u$ and $v$ are the velocity components along the $x$-direction and $y$ directions respectively. The physical configuration of the problem is as shown in Fig. 1.

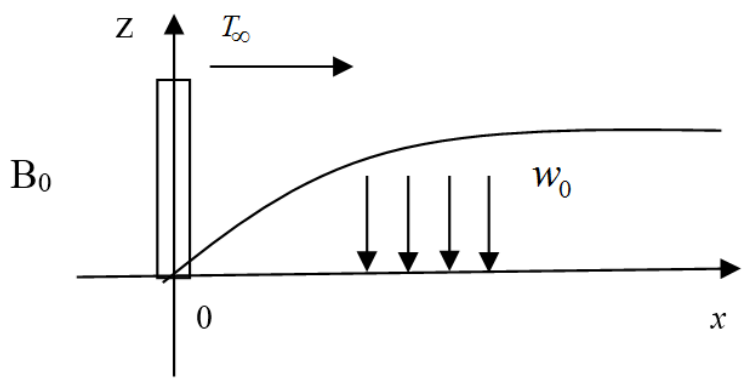

Figure 1. Physical configuration of the problem

The governing equations for the unsteady MHD free convection flow of an incompressible viscous electrically conducting fluid with simultaneous heat and mass transfer over an infinite vertical plate through porous medium under the influence of uniform transverse magnetic field with respect to the frame are given by

Equation of continuity:

$$
\frac{\partial u}{\partial x}+\frac{\partial v}{\partial y}=0
$$

Momentum equation:

$$
\begin{aligned}
\frac{\partial u}{\partial t}-w \frac{\partial u}{\partial z} & =v \frac{\partial^{2} u}{\partial z^{2}}+\frac{\alpha_{1}}{\rho} \frac{\partial^{3} u}{\partial z^{2} \partial t}+\frac{B_{0} J_{y}}{\rho} \\
& -\frac{v}{K(t)} u+g \beta\left(T-T_{\infty}\right)+g \beta^{*}\left(C-C_{\infty}\right)
\end{aligned}
$$

$\frac{\partial v}{\partial t}-w \frac{\partial v}{\partial z}=v \frac{\partial^{2} v}{\partial z^{2}}+\frac{\alpha_{1}}{\rho} \frac{\partial^{3} v}{\partial z^{2} \partial t}-\frac{B_{0} J_{x}}{\rho}-\frac{v}{K(t)} v$

Equation of energy:

$$
\frac{\partial T}{\partial t}-w \frac{\partial T}{\partial z}=\alpha \frac{\partial^{2} T}{\partial z^{2}}-S_{1}\left(T-T_{\infty}\right)
$$

Equation of concentration

$$
\frac{\partial C}{\partial t}-w \frac{\partial C}{\partial z}=D \frac{\partial^{2} C}{\partial z^{2}}-K_{1}\left(C-C_{\infty}\right)
$$

When the strength of the magnetic field is very large, the generalized ohm's law is modified to include the hall current so that

$$
J+\frac{\omega_{e} \tau_{e}}{B_{O}}(J \times B)=\sigma\left\lfloor E+V \times B+\frac{1}{e \eta_{e}} \nabla P_{e}\right\rfloor
$$

The ion-slip and thermo electric effects are not included in equation (6) Further it is assumed that $\omega_{e} \tau_{e} \sim 0$ (1) and $\omega_{i} \tau_{i}<<1$, In the equation (6) the electron pressure gradient, the ion-slip and thermo-electric effects are neglected. We also assume that the electric field $E=0$ under assumptions reduces to

$$
\begin{aligned}
& J_{x}+m J_{y}=\sigma B_{0} v \\
& J_{y}-m J_{x}=-\sigma B_{0} u
\end{aligned}
$$

Where $m=\tau_{\mathrm{e}} \omega_{e}$ is the hall parameter.

On solving equations (7) and (8) we obtain

$$
\begin{aligned}
& J_{x}=\frac{\sigma B_{0}}{1+m^{2}}(v+m u) \\
& J_{y}=\frac{\sigma B_{0}}{1+m^{2}}(m v-u)
\end{aligned}
$$

Substituting the equations (9) and (10) in (3) and (2) respectively, we obtain

$$
\begin{aligned}
& \frac{\partial u}{\partial t}-w \frac{\partial u}{\partial z}=v \frac{\partial^{2} u}{\partial z^{2}}+\frac{\alpha_{1}}{\rho} \frac{\partial^{3} u}{\partial z^{2} \partial t} \\
&+\left(\frac{\sigma B_{0}^{2}}{1+m^{2}}(m v-u)-\frac{v}{K(t)}\right) u+g \beta\left(T-T_{\infty}\right)+g \beta^{*}\left(C-C_{\infty}\right) \\
& \frac{\partial v}{\partial t}-w \frac{\partial v}{\partial z}= v \frac{\partial^{2} v}{\partial z^{2}}+\frac{\alpha_{1}}{\rho} \frac{\partial^{3} v}{\partial z^{2} \partial t} \\
&-\left(\frac{\sigma B_{0}^{2}}{1+m^{2}}(v+m u)+\frac{v}{K(t)}\right) v
\end{aligned}
$$

Combining equations (11) and (12), Let $q=u+i v$

$$
\begin{aligned}
\frac{\partial q}{\partial t} & -w \frac{\partial q}{\partial z}=v \frac{\partial^{2} q}{\partial z^{2}}+\frac{\alpha_{1}}{\rho} \frac{\partial^{3} q}{\partial z^{2} \partial t} \\
& -\left(\frac{\sigma B_{0}^{2}}{1+m^{2}}+\frac{v}{K(t)}\right) q+g \beta\left(T-T_{\infty}\right)+g \beta^{*}\left(C-C_{\infty}\right)
\end{aligned}
$$


The boundary conditions are

$$
\begin{aligned}
q(z, t)=T(z, t)=C(z, t) & =f(t) & \text { at } & z=0 \\
& =0 & \text { at } & z \rightarrow \infty
\end{aligned}
$$

Where, $f(t)=1+\varepsilon e^{i \omega t}$ With foregoing assumptions and taking usual Boussinesq's approximation into account as well as the following non-dimensional variables.

$$
\begin{aligned}
q^{*} & =\frac{q}{w_{0}}, z=\frac{w_{0} z^{*}}{v}, t^{*}=\frac{w_{0}^{2} t}{v}, \omega^{*} \\
& =\frac{v \omega}{w_{0}{ }^{2}}, \theta=\frac{T-T_{\infty}}{T_{w}-T_{\infty}}, \phi=\frac{C-C_{\infty}}{C_{w}-C_{\infty}}
\end{aligned}
$$

Making use of non-dimensional variables, the governing equations reduces to (Dropping asterisks)

$$
\begin{aligned}
\frac{\partial q}{\partial t}-w_{o}\left(1+\varepsilon e^{i \omega t}\right) & \frac{\partial q}{\partial y}=\frac{\partial^{2} q}{\partial y^{2}}+\alpha \frac{\partial^{3} q}{\partial z^{2} \partial t} \\
& -\left(\frac{M^{2}}{1+m^{2}}+\frac{1}{K\left(1+\varepsilon e^{i \omega t}\right)}\right) q+\operatorname{Gr} \theta+\operatorname{Gm} C
\end{aligned}
$$

$\operatorname{Pr} \frac{\partial \theta}{\partial t}-w_{0}\left(1+\varepsilon e^{i \omega t}\right) \operatorname{Pr} \frac{\partial \theta}{\partial z}=\frac{\partial^{2} \theta}{\partial z^{2}}-\operatorname{Pr} S \theta$

$\operatorname{Sc} \frac{\partial \phi}{\partial t}-w_{0}\left(1+\varepsilon e^{i \omega t}\right) \operatorname{Sc} \frac{\partial \phi}{\partial z}=\frac{\partial^{2} \phi}{\partial z^{2}}-\operatorname{KcSc} \phi$,

The corresponding boundary conditions are

$$
\begin{aligned}
& q(z, t)=\theta(z, t)=\phi(z, t)=1+\varepsilon e^{i \omega t} \quad \text { at } \quad z=0 \\
& =0 \quad \text { at } \quad z \rightarrow \infty
\end{aligned}
$$

Where, $M^{2}=\frac{\sigma B_{0}^{2} v}{\rho w_{0}^{2}}$ is the Hartmann number (Magnetic field parameter), $K=\frac{v^{2}}{k w_{0}^{2}}$ is the permeability parameter (Porosity or Darcy parameter), $\alpha=\frac{\alpha_{1} w_{0}^{2}}{\rho v^{2}}$ is the second grade fluid parameter, $\operatorname{Pr}=\frac{v}{\alpha}$ is the Prandtl number, $\mathrm{Sc}=\frac{v}{D}$ is the Schmidt number, $\mathrm{Kc}=\frac{K_{1} v}{w_{0}^{2}}$ is the chemical reaction parameter, $S=\frac{S_{1} v}{w_{0}^{2}}$ is the Heat Source parameter, $\mathrm{Gr}=\frac{g \beta v\left(T_{w}-T_{\infty}\right)}{w_{0}^{3}}$ is the thermal Grashof number and $\mathrm{Gm}=\frac{g \beta^{*} v\left(C_{w}-C_{\infty}\right)}{w_{0}^{3}}$ is the mass Grashof number.
In order to solve the equations, (15) - (17) using boundary conditions (18), we assume the solutions of the following form, because the amplitude $\varepsilon(<<1)$ of permeability is very small.

$$
\begin{aligned}
& q(z, t)=q_{0}(z)+\varepsilon q_{1}(z) e^{i \omega t} \\
& \theta(z, t)=\theta_{0}(z)+\varepsilon \theta_{1}(z) e^{i \omega t} \\
& \phi(z, t)=\phi_{0}(z)+\varepsilon \phi_{1}(z) e^{i \omega t}
\end{aligned}
$$

Substituting the Eqs. (12) - (14) into the Eqs. (8) - (10) respectively and equate the harmonic and non-hormonic terms to obtain the zeroth and first orders ordinary differential equations for momentum, temperature and concentration distributions.

The skin friction at the plate in terms of amplitude and phase is given by

$$
\begin{aligned}
\tau=\left(\frac{\partial q}{\partial z}\right)_{z=0} & =\left(\frac{\partial q_{0}}{\partial z}\right)_{z=0}+\varepsilon\left(\frac{\partial q_{1}}{\partial z}\right)_{z=0} \\
& =F_{1}+\varepsilon\left|F_{2}\right| \cos (\omega t+\psi)
\end{aligned}
$$

Where, $F_{1}=\left(\frac{\partial q_{0}}{\partial z}\right)_{z=0} ; F_{2}=\left(\frac{\partial q_{1}}{\partial z}\right)_{z=0}$ and $\tan (\psi)=\frac{\operatorname{Re}\left[F_{2}\right]}{\operatorname{Im}\left[F_{2}\right]}$.

The Nusselt number at the plate in terms of amplitude and phase is given by

$$
\begin{aligned}
N u=-\left(\frac{\partial \theta}{\partial z}\right)_{z=0} & =\left(\frac{\partial \theta_{0}}{\partial z}\right)_{z=0}+\varepsilon\left(\frac{\partial \theta_{1}}{\partial z}\right)_{z=0} \\
& =F_{3}+\varepsilon\left|F_{4}\right| \cos (\omega t+\gamma)
\end{aligned}
$$

Where, $F_{3}=\left(\frac{\partial \theta_{0}}{\partial z}\right)_{z=0} ; F_{4}=\left(\frac{\partial \theta_{1}}{\partial z}\right)_{z=0}$ and $\tan (\gamma)=\frac{\operatorname{Re}\left[F_{4}\right]}{\operatorname{Im}\left[F_{4}\right]}$.

The Sherwood number at the plate in terms of amplitude and phase is given by

$$
\begin{aligned}
S h=-\left(\frac{\partial \phi}{\partial z}\right)_{z=0} & =\left(\frac{\partial \phi_{0}}{\partial z}\right)_{z=0}+\varepsilon\left(\frac{\partial \phi_{1}}{\partial z}\right)_{z=0} \\
& =F_{5}+\varepsilon\left|F_{6}\right| \cos (\omega t+\zeta)
\end{aligned}
$$

Where, $F_{5}=\left(\frac{\partial \phi_{0}}{\partial z}\right)_{z=0} ; F_{6}=\left(\frac{\partial \phi_{1}}{\partial z}\right)_{z=0}$ and $\tan (\zeta)=\frac{\operatorname{Re}\left[F_{6}\right]}{\operatorname{Im}\left[F_{6}\right]}$.

\section{RESULTS AND DISCUSSION:}

The governing equations of the flow field are solved by a regular perturbation method for small amplitude of the permeability. The closed form solutions for the velocity, temperature and concentration have been derived analytically and also its behavior is computationally discussed with reference to different flow parameters. Figures (2-5) represent velocity, Figures (6) and Figures (7) represent the temperature 
and concentration distributions respectively. The stresses, Nusselt number and Sherwood number at the plate are evaluated numerically and discussed with governing parameters and are tabulated in the tables (1-3).

From the Figures (2-4), we noticed that the magnitude of the velocity component $u$ reduces with increasing the intensity of the magnetic field $\mathrm{M}$, Whereas the velocity component $u$ enhance with increasing permeability parameter $\mathrm{K}$, thermal Grashof number Gr or mass Grashof number Gm throughout the fluid region. Lower the permeability of the porous medium lesser the fluid speed in the entire region. The magnitude of the velocity component $v$ is reduces with $\mathrm{M}$ and $\mathrm{K}$ but it initially reduces and then gradually enhances with $\mathrm{Pr}$ and $\mathrm{S}$. The reversal behaviour is observed with increasing $\mathrm{Gr}$ and $\mathrm{Gm}$.

The Figures (4-5) depict the velocity component $u$ experiences retardation in the flow field with increasing the chemical reaction parameter $\mathrm{Kc}$, mass Grashof number $\mathrm{Gm}$.

Figures 6 (a-d) showed the effect of Heat source parameter $S$, the Prandtl number Pr, suction velocity $v_{0}$ and the frequency of oscillation $\omega$ on the temperature of the flow field. We noted that the temperature of the flow field diminishes as the Prandtl number increases. This is consistent with the fact that the thermal boundary layer thickness decreases with increasing Prandtl number. With increasing heat source parameter reduces the temperature of the flow field. This may happen due the elastic property of the fluid. It is observed that temperature of the flow field diminishes as the suction parameter or the frequency of oscillation increases.

Figures 7(a-d) depict the effect of the Schmidt number Sc and the frequency of oscillation $\omega$ on concentration distribution. The concentration distribution decreases at all points of the flow field with the increase in the Schmidt number Sc or chemical reaction parameter Kc. This shows that the heavier diffusing species have a greater retarding effect on the concentration distribution of the flow field. Also, it is observed that presence of the frequency of oscillation $\omega$ or increasing the suction velocity reduces the concentration distribution.
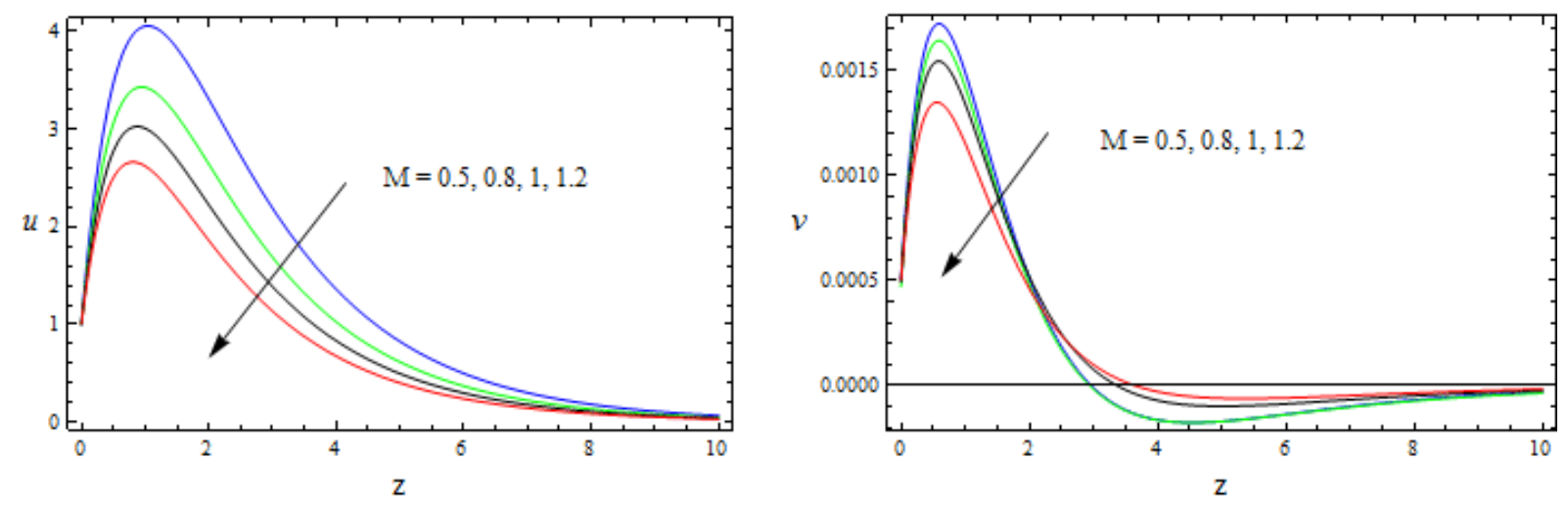

Figure 2. The velocity Profiles for $u$ and $v$ against $\mathrm{M}$ with $\mathrm{K}=1, \mathrm{~m}=1, \alpha=1, \mathrm{Pr}=0.71, \mathrm{~S}=1, \mathrm{Sc}=0.22, \mathrm{Kc}=1, \mathrm{Gr}=5, \mathrm{Gm}=10, w_{0}=0.2$ and $\omega=\pi / 6$
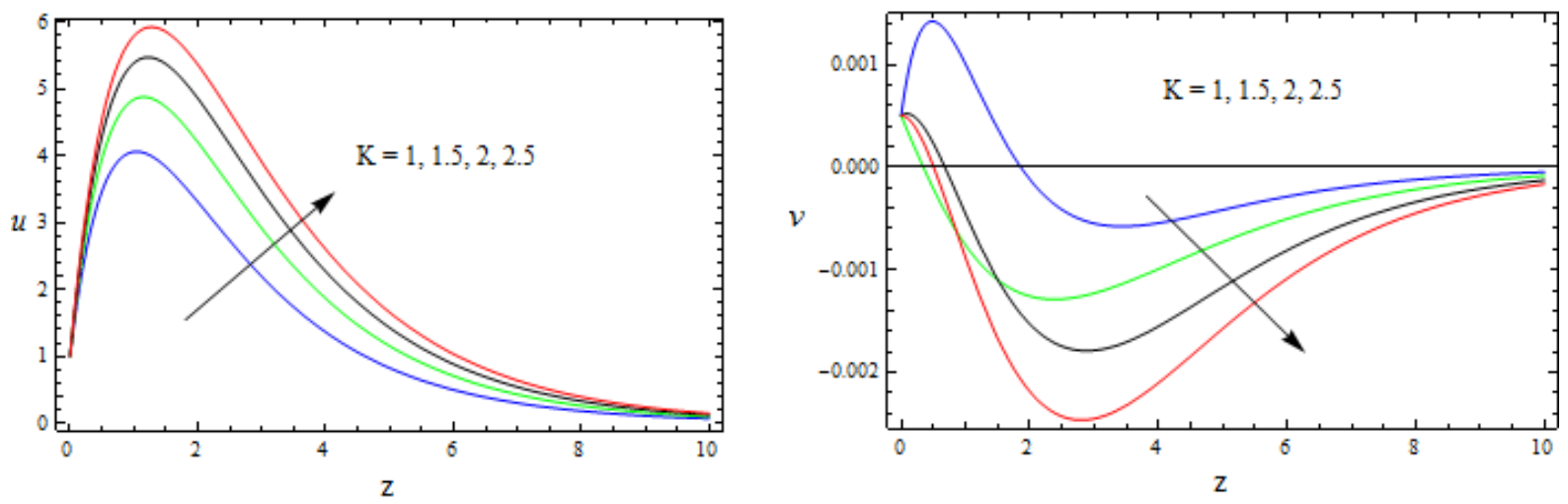

Figure 3. The velocity Profiles for $u$ and $v$ against $K$ with

$\mathrm{M}=2, \mathrm{~m}=1, \alpha=1, \mathrm{Pr}=0.71, \mathrm{~S}=1, \mathrm{Sc}=0.22, \mathrm{Kc}=1, \mathrm{Gr}=5, \mathrm{Gm}=10, w_{0}=0.2$ and $\omega=\pi / 6$ 
International Journal of Applied Engineering Research ISSN 0973-4562 Volume 13, Number 7 (2018) pp. $4782-4790$

(C) Research India Publications. https://dx.doi.org/10.37622/IJAER/13.7.2018.4782-4790
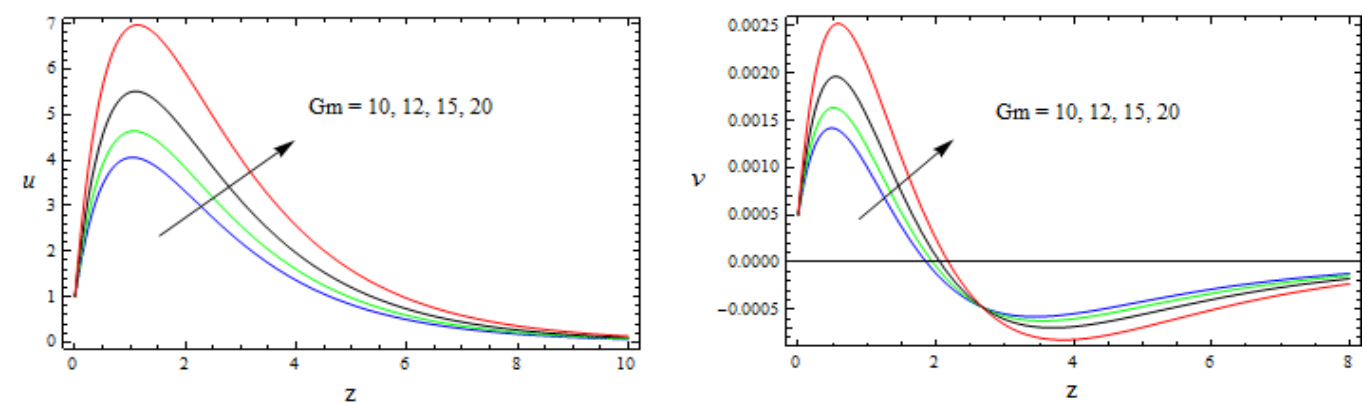

Figure 4. The velocity Profiles for $u$ and $v$ against Gm with $\mathrm{M}=2, \mathrm{~m}=1, \alpha=1, \mathrm{~K}=1, \mathrm{Pr}=0.71, \mathrm{~S}=1, \mathrm{Sc}=0.22, \mathrm{Kc}=1, \mathrm{Gr}=5, w_{0}=0.2$ and $\omega=\pi / 6$
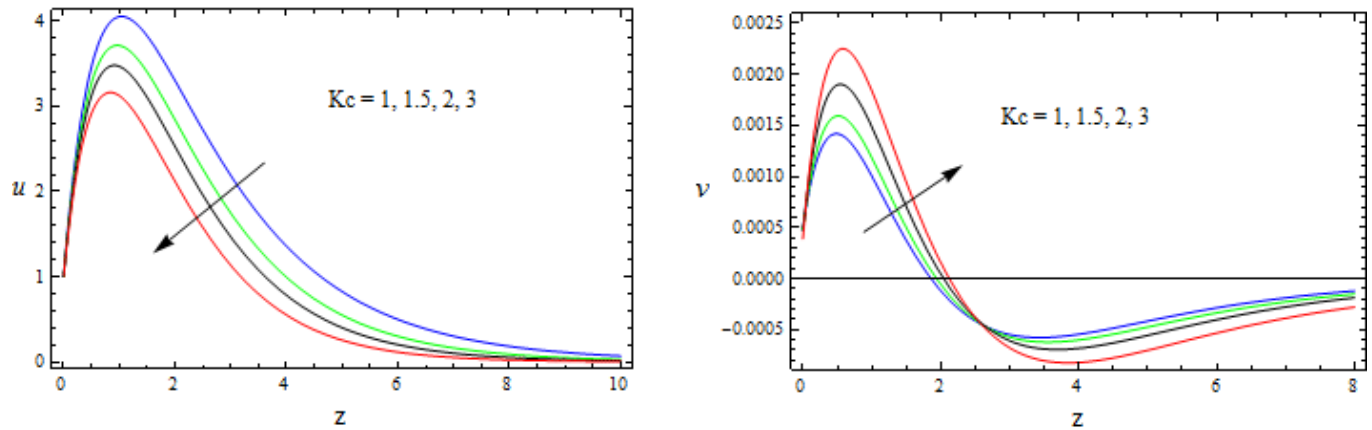

Figure 5. The velocity Profiles for $u$ and $v$ against Kc with $\mathrm{M}=2, \mathrm{~m}=1, \alpha=1, \mathrm{~K}=1, \mathrm{Pr}=0.71, \mathrm{~S}=1, \mathrm{Sc}=0.22, \mathrm{Gr}=5, \mathrm{Gm}=10, w_{0}=0.2$ and $\omega=\pi / 6$

The skin friction, Nusselt number and Sherwood number are calculated numerically and tabulated in the tables (1-3). The skin friction increases with the increase in $K, \mathrm{Gr}, \mathrm{Gm}, \alpha, m$ and $v_{0}$ and decreases with the increase in $M, \operatorname{Pr}, \mathrm{Sc}, \mathrm{Kc}, S$ and $\omega$ (Table. 1). Nusselt number $(\mathrm{Nu})$ at the surface of the plate and amplitude increase with increase $S, \operatorname{Pr}$ and $v_{0}$. Also enhance the amplitude, the rate of heat transfer decrease the phase angle with increase the frequency of oscillations $\omega$ (Table. 2). Schmidt number Sc, chemical reaction parameter $\mathrm{Kc}$, the frequency of oscillations $\omega$ and suction parameter $v_{0}$ increase the amplitude, the phase angle the rate of mass transfer at the surface of the plate (Table. 3 ).
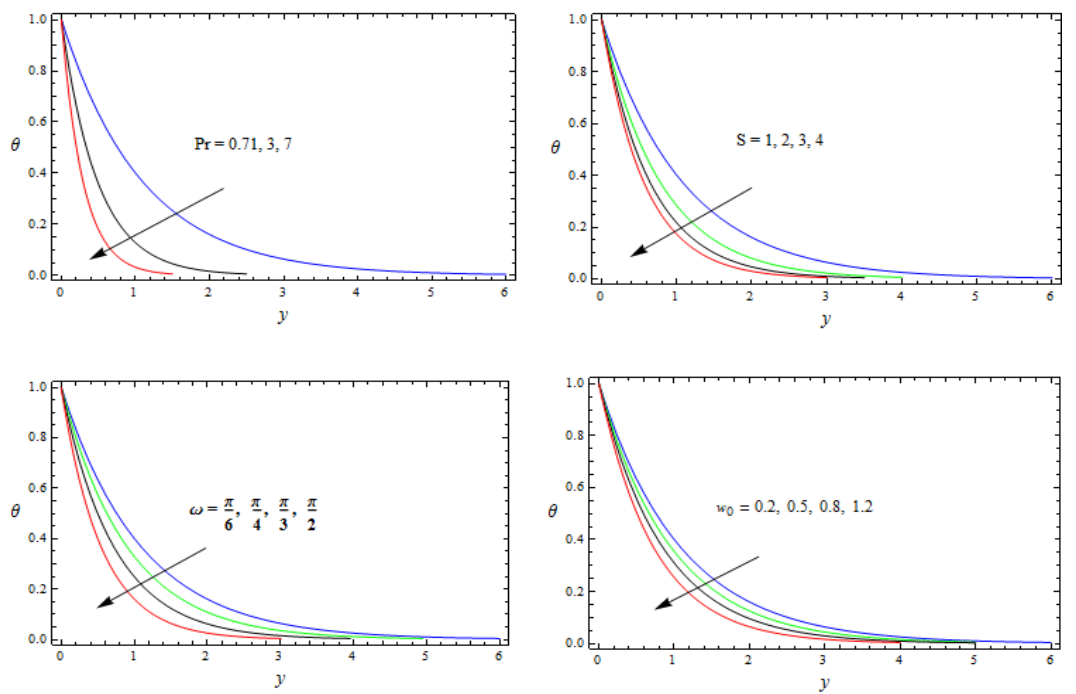

Figure 6(a-d): The temperature profiles against $\operatorname{Pr}, \mathrm{S}, \omega$ and $w_{0}$ with $\varepsilon=0.001, t=0.2$ 
International Journal of Applied Engineering Research ISSN 0973-4562 Volume 13, Number 7 (2018) pp. $4782-4790$

(C) Research India Publications. https://dx.doi.org/10.37622/IJAER/13.7.2018.4782-4790
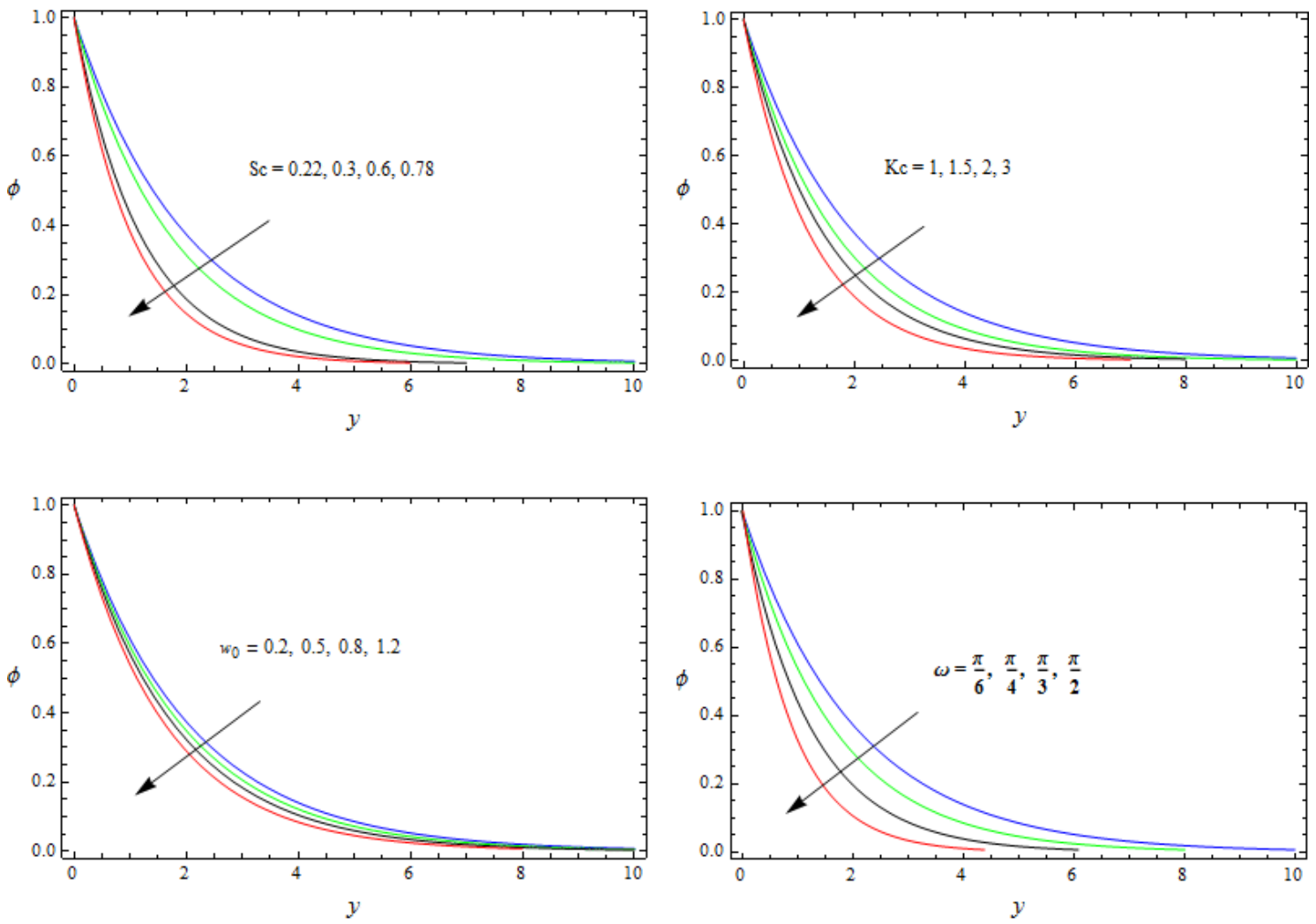

Figure 7(a-d): The Concentration profiles against Sc, Kc, $w_{0}$ and $\omega$ with $\varepsilon=0.001, t=0.2$

Table 1. Shear stresses with $\varepsilon=0.001, t=0.2$

\begin{tabular}{|c|c|c|c|c|c|c|c|c|c|c|c|c|c|c|}
\hline M & $\mathrm{K}$ & $\operatorname{Pr}$ & $\alpha$ & $m$ & $\mathrm{Sc}$ & $\mathrm{Kc}$ & $S$ & $\mathrm{Gr}$ & $\mathrm{Gm}$ & $w_{0}$ & $\omega$ & $\begin{array}{l}\text { Amplitude } \\
\qquad\left|F_{2}\right|\end{array}$ & $\begin{array}{r}\text { Phase } \\
\text { Angle } \\
(\psi)\end{array}$ & $\tau$ \\
\hline 0.5 & 2 & 0.71 & 1 & 1 & 0.22 & 1 & 1 & 5 & 10 & 0.2 & $\pi / 6$ & 11.6855 & -1.08748 & 9.91855 \\
\hline 2 & 2 & 0.71 & 1 & 1 & 0.22 & 1 & 1 & 5 & 10 & 0.2 & $\pi / 6$ & 4.22966 & -1.554741 & 3.45748 \\
\hline 3 & 2 & 0.71 & 1 & 1 & 0.22 & 1 & 1 & 5 & 10 & 0.2 & $\pi / 6$ & 1.29856 & -1.41152 & 0.97856 \\
\hline 2 & 3 & 0.71 & 1 & 1 & 0.22 & 1 & 1 & 5 & 10 & 0.2 & $\pi / 6$ & 12.9847 & -1.00211 & 10.8633 \\
\hline 2 & 4 & 0.71 & 1 & 1 & 0.22 & 1 & 1 & 5 & 10 & 0.2 & $\pi / 6$ & 13.1455 & -0.92522 & 11.5585 \\
\hline 2 & 2 & 3 & 1 & 1 & 0.22 & 1 & 1 & 5 & 10 & 0.2 & $\pi / 6$ & 10.9614 & -1.22366 & 8.71415 \\
\hline 2 & 2 & 7 & 1 & 1 & 0.22 & 1 & 1 & 5 & 10 & 0.2 & $\pi / 6$ & 10.8785 & -1.30847 & 8.13415 \\
\hline 2 & 2 & 7 & 1 & 2 & 0.22 & 1 & 1 & 5 & 10 & 0.2 & $\pi / 6$ & 10.9558 & -1.24552 & 11.2556 \\
\hline 2 & 2 & 7 & 1 & 3 & 0.22 & 1 & 1 & 5 & 10 & 0.2 & $\pi / 6$ & 10.8859 & -1.52214 & 12.0895 \\
\hline 2 & 2 & 0.71 & 2 & 1 & 0.22 & 1 & 1 & 5 & 10 & 0.2 & $\pi / 6$ & 12.2785 & -1.00352 & 10.2856 \\
\hline 2 & 2 & 0.71 & 3 & 1 & 0.22 & 1 & 1 & 5 & 10 & 0.2 & $\pi / 6$ & 13.8522 & -0.93415 & 11.3859 \\
\hline 2 & 2 & 0.71 & 1 & 1 & 0.3 & 1 & 1 & 5 & 10 & 0.2 & $\pi / 6$ & 13.2102 & -1.05263 & 9.40145 \\
\hline 2 & 2 & 0.71 & 1 & 1 & 0.6 & 1 & 1 & 5 & 10 & 0.2 & $\pi / 6$ & 13.5633 & -0.09585 & 8.21252 \\
\hline 2 & 2 & 0.71 & 1 & 1 & 0.22 & 1.5 & 1 & 5 & 10 & 0.2 & $\pi / 6$ & 13.0100 & -0.99695 & 9.30663 \\
\hline 2 & 2 & 0.71 & 1 & 1 & 0.22 & 2 & 1 & 5 & 10 & 0.2 & $\pi / 6$ & 14.0411 & -0.85525 & 8.86415 \\
\hline 2 & 2 & 0.71 & 1 & 1 & 0.22 & 1 & 2 & 5 & 10 & 0.2 & $\pi / 6$ & 11.6255 & -1.13496 & 9.61255 \\
\hline 2 & 2 & 0.71 & 1 & 1 & 0.22 & 1 & 3 & 5 & 10 & 0.2 & $\pi / 6$ & 11.3855 & -1.18452 & 9.4074 \\
\hline 2 & 2 & 0.71 & 1 & 1 & 0.22 & 1 & 1 & 8 & 10 & 0.2 & $\pi / 6$ & 12.8412 & -0.98566 & 11.6225 \\
\hline
\end{tabular}


International Journal of Applied Engineering Research ISSN 0973-4562 Volume 13, Number 7 (2018) pp. $4782-4790$

(C) Research India Publications. https://dx.doi.org/10.37622/IJAER/13.7.2018.4782-4790

\begin{tabular}{|l|l|l|l|l|l|l|l|l|l|l|l|l|l|l|}
\hline 2 & 2 & 0.71 & 1 & 1 & 0.22 & 1 & 1 & $\mathbf{1 0}$ & 10 & 0.2 & $\pi / 6$ & 13.7110 & -0.92966 & 12.8796 \\
\hline 2 & 2 & 0.71 & 1 & 1 & 0.22 & 1 & 1 & 5 & $\mathbf{1 5}$ & 0.2 & $\pi / 6$ & 16.7522 & -1.17958 & 13.8752 \\
\hline 2 & 2 & 0.71 & 1 & 1 & 0.22 & 1 & 1 & 5 & $\mathbf{2 0}$ & 0.2 & $\pi / 6$ & 21.9966 & -1.22985 & 17.8514 \\
\hline 2 & 2 & 0.71 & 1 & 1 & 0.22 & 1 & 1 & 5 & 10 & $\mathbf{0 . 5}$ & $\pi / 6$ & 23.0452 & -0.60411 & 10.0263 \\
\hline 2 & 2 & 0.71 & 1 & 1 & 0.22 & 1 & 1 & 5 & 10 & $\mathbf{0 . 8}$ & $\pi / 6$ & 33.0255 & -0.02014 & 10.5198 \\
\hline 2 & 2 & 0.71 & 1 & 1 & 0.22 & 1 & 1 & 5 & 10 & 0.2 & $\pi / 4$ & 8.03522 & -0.88652 & 9.91301 \\
\hline 2 & 2 & 0.71 & 1 & 1 & 0.22 & 1 & 1 & 5 & 10 & 0.2 & $\pi / 3$ & 6.43936 & -0.63128 & 9.91102 \\
\hline 2 & 2 & 0.71 & 1 & 1 & 0.22 & 1 & 1 & 5 & 10 & 0.2 & $\pi / 2$ & 5.48966 & -0.27396 & 9.91639 \\
\hline
\end{tabular}

Table 2. Nusselt number (Nu) with $\varepsilon=0.001, t=0.2$

\begin{tabular}{|c|c|c|c|c|c|c|}
\hline Pr & $S$ & $w_{0}$ & $\omega$ & $\begin{array}{c}\text { Amplitude } \\
\left|F_{4}\right|\end{array}$ & $\begin{array}{c}\text { Phase } \\
\text { Angle } \gamma\end{array}$ & $\mathrm{Nu}$ \\
\hline $\mathbf{0 . 7 1}$ & $\mathbf{1}$ & $\mathbf{0 . 2}$ & $\pi / 6$ & 1.00365 & 1.39869 & 0.91666 \\
\hline $\mathbf{3}$ & 1 & 0.2 & $\pi / 6$ & 2.20629 & 1.44928 & 2.05788 \\
\hline $\mathbf{7}$ & 1 & 0.2 & $\pi / 6$ & 3.60564 & 1.47239 & 3.43676 \\
\hline 0.71 & $\mathbf{2}$ & 0.2 & $\pi / 6$ & 1.31588 & 1.48355 & 1.26473 \\
\hline 0.71 & $\mathbf{3}$ & 0.2 & $\pi / 6$ & 1.56988 & 1.51423 & 1.53210 \\
\hline 0.71 & 1 & $\mathbf{0 . 5}$ & $\pi / 6$ & 1.10256 & 1.46078 & 1.03861 \\
\hline 0.71 & 1 & $\mathbf{0 . 8}$ & $\pi / 6$ & 1.21656 & 1.48335 & 1.17317 \\
\hline 0.71 & 1 & 0.2 & $\pi / 4$ & 1.06122 & 1.31218 & 0.91670 \\
\hline 0.71 & 1 & 0.2 & $\pi / 3$ & 1.12272 & 1.24229 & 0.91673 \\
\hline
\end{tabular}

Table 3. Sherwood number (Sh) with $\varepsilon=0.001, t=0.2$

\begin{tabular}{|c|c|c|c|c|c|c|}
\hline Sc & Kc & $w_{0}$ & $\omega$ & $\begin{array}{c}\text { Amplitude } \\
\left|F_{6}\right|\end{array}$ & $\begin{array}{c}\text { Phase } \\
\text { Angle }(\zeta)\end{array}$ & Sh \\
\hline $\mathbf{0 . 2 2}$ & $\mathbf{1}$ & $\mathbf{0 . 2}$ & $\pi / 6$ & 0.536249 & 1.365880 & 0.491611 \\
\hline $\mathbf{0 . 3}$ & 1 & 0.2 & $\pi / 6$ & 0.632410 & 1.373015 & 0.578602 \\
\hline $\mathbf{0 . 6}$ & 1 & 0.2 & $\pi / 6$ & 0.916662 & 1.393062 & 0.836984 \\
\hline 0.22 & $\mathbf{1 . 5}$ & 0.2 & $\pi / 6$ & 0.629483 & 1.429490 & 0.596922 \\
\hline 0.22 & $\mathbf{2}$ & 0.2 & $\pi / 6$ & 0.712364 & 1.464392 & 0.685691 \\
\hline 0.22 & 1 & $\mathbf{0 . 5}$ & $\pi / 6$ & 0.574058 & 1.422405 & 0.527289 \\
\hline 0.22 & 1 & $\mathbf{0 . 8}$ & $\pi / 6$ & 0.603609 & 1.454160 & 0.565233 \\
\hline 0.22 & 1 & 0.2 & $\pi / 4$ & 0.566008 & 1.276890 & 0.491634 \\
\hline 0.22 & 1 & 0.2 & $\pi / 3$ & 0.599008 & 1.206869 & 0.491650 \\
\hline
\end{tabular}


Table 4. Comparison of Results

\begin{tabular}{|c|c|c|c|c|}
\hline Sc & $w_{0}$ & $\omega$ & Sh & \\
\hline & & & Ashraf et al.[33] & Present work [Kc=0] \\
\hline $\mathbf{0 . 2 2}$ & $\mathbf{0 . 2}$ & $\pi / 6$ & 0.045262 & 0.044020 \\
\hline $\mathbf{0 . 3}$ & 0.2 & $\pi / 6$ & 0.061854 & 0.060238 \\
\hline $\mathbf{0 . 6}$ & 0.2 & $\pi / 6$ & 0.120552 & 0.120325 \\
\hline 0.22 & $\mathbf{0 . 5}$ & $\pi / 6$ & 0.115245 & 0.110179 \\
\hline 0.22 & $\mathbf{0 . 8}$ & $\pi / 6$ & 0.176334 & 0.176133 \\
\hline 0.22 & 0.2 & $\pi / 4$ & 0.046625 & 0.044236 \\
\hline 0.22 & 0.2 & $\pi / 3$ & 0.046246 & 0.044253 \\
\hline
\end{tabular}

\section{CONCLUSIONS}

1. The velocity reduces with increasing the intensity of the magnetic field or Prandtl number Pr or Heat source parameter $S$.

2. The velocity enhance with increasing thermal Grashof number $\mathrm{Gr}$ or mass Grashof number or second grade fluid parameter $\alpha$ or Hall parameter $m$.

3. The resultant velocity enhances with increasing the permeability parameter $\mathrm{K}$ throughout the fluid region. Lower the permeability of the porous medium lesser the fluid speed in the entire region.

4. The magnitude of the temperature of the flow field diminishes as the Prandtl number, Heat source parameter $S$ or suction parameter $w_{0}$ or the frequency of oscillation.

5. The concentration reduces at all points of the flow field with the increase in the Schmidt number Sc, chemical reaction parameter $\mathrm{Kc}$, suction parameter $w_{0}$ and presence of the frequency of oscillation $\omega$.

6. The skin friction increases with the increase in $K \mathrm{Gr}$, $\mathrm{Gm}, m$ and $w_{0}$ and decreases with the increase in $M$, Pr, Sc, Kc, $S$ and $\omega$.

7. Nusselt number $(\mathrm{Nu})$ at the surface of the plate and amplitude increase with increase $S$, Pr and $w_{0}$. Also enhance the amplitude, the rate of heat transfer decrease the phase angle with increase the frequency of oscillations $\omega$.

8. Schmidt number $\mathrm{Sc}$, chemical reaction parameter $\mathrm{Kc}$, the frequency of oscillations $\omega$ and suction parameter $w_{0}$ increase the amplitude, the phase angle the rate of mass transfer at the surface of the plate.

9. Increase the frequency of oscillations $\omega$, enhance the amplitude, the rate of mass transfer decrease the phase angle.

\section{REFERENCES}

[1]. R.K. Deka. Hall effects on MHD flow past an accelerated plate, Theoret. Appl. Mech. 35(4) (2008):333-346.

[2]. S.K. Ghosh, O.A. Beg, and M. Narahari. Hall effects on MHD flow in a rotating system with heat transfer characteristics, Meccanica 44 (2009):741-765.

[3]. D.V. Krishna, D.R.V. Prasada Rao and A.S. Ramachandra Murthy. Hydromagnetic convection flow through a porous medium in a rotating channel, J. of Engng. Phys. And Thermophysics 75(2) (2002):281291.

[4]. D.S. Chauhan and R. Agrawal. Effects of Hall current on MHD flow in a rotating channel partially filled with a porous medium, Chemical Engineering Communications 197(6) (2010):830-845.

[5]. D.S. Chauhan and R. Agrawal. Effects of hall current on MHD Couette flow in a channel partially filled with a porous medium in a rotating system, Meccanica 47(2012):405-421.

[6]. Dileep Singh Chauhan, Priyanka Rastogi, "Hall Effects on MHD Slip Flow and Heat Transfer Through a Porous Medium over an Accelerated Plate in a Rotating System," International Journal of Nonlinear Science, 14(2) (2012): 228-236.

[7]. M.VeeraKrishna and J.Prakash, "Hall Current Effects on Unsteady MHD Flow in a Rotating Parallel Plate Channel Bounded by Porous Bed on the Lower HalfDarcy Lapwood Model, Open Journal of Fluid Dynamics, 5 (2015):275-294

[8]. Ashaf A. Moniem, W.S.Hassanin, Solution of MHD flow past a vertical porous plate through a porous medium under oscillatory suction," Applied Mathematics, 4, (2013), 694-702.

[9]. B.Lakshmanna; S.Venkateswarlu, "Heat and Mass Transfer on MHD Flow of an incompressible fluid past an infinite vertical porous plate", Journal of Ultra 
International Journal of Applied Engineering Research ISSN 0973-4562 Volume 13, Number 7 (2018) pp. $4782-4790$

(C) Research India Publications. https://dx.doi.org/10.37622/IJAER/13.7.2018.4782-4790

Scientist of Physical Sciences, Volume 29, Issue 9, Page number 234-247,2017.

[10]. M.Veera Krishna, M.Gangadhar Reddy, MHD Free Convective Boundary Layer Flow through Porous medium Past a Moving Vertical Plate with Heat Source and Chemical Reaction,Materials Today: Proceedings, vol. 5, pp.91-98, 2018. https://doi.org/10.1016/j.matpr.2017.11.058.

[11]. M.Veera Krishna, G.Subba Reddy, MHD Forced Convective flow of Non-Newtonian fluid through Stumpy Permeable Porous medium, Materials Today: Proceedings, vol. 5, pp. 175-183, 2018. https://doi.org/10.1016/j.matpr.2017.11.069.

[12]. M.Veera Krishna, Kamboji Jyotghi, Hall effects on MHD Rotating flow of a Visco-elastic Fluid through a Porous medium Over an Infinite Oscillating Porous Plate with Heat source and Chemical reaction, Materials Today: Proceedings, vol. 5, pp. 367-380, 2018. https://doi.org/10.1016/j.matpr.2017.11.094.

[13]. B. Siva Kumar Reddy, M. Veera Krishna, K.V.S.N. Rao, R. Bhuvana Vijaya, HAM Solutions on MHD flow of nano-fluid through saturated porous medium with Hall effects, Materials Today: Proceedings, vol. 5, pp. 120-131, 2018. https://doi.org/10.1016/j.matpr.2017.11.062.

[14]. VeeraKrishna.M and B.V.Swarnalathamma, Convective Heat and Mass Transfer on MHD Peristaltic Flow of Williamson Fluid with the Effect of Inclined Magnetic Field," AIP Conference Proceedings, vol. 1728, p. 020461, 2016. DOI: $10.1063 / 1.4946512$

[15]. Swarnalathamma. B. V. and M. Veera Krishna, Peristaltic hemodynamic flow of couple stress fluid through a porous medium under the influence of magnetic field with slip effect AIP Conference Proceedings, vol. 1728, p. 020603, 2016. DOI: 10.1063/1.4946654.

[16]. VeeraKrishna.M and M.Gangadhar Reddy MHD free convective rotating flow of Visco-elastic fluid past an infinite vertical oscillating porous plate with chemical reaction, IOP Conf. Series: Materials Science and Engineering, vol. 149, p. 012217, 2016 DOI: 10.1088/1757-899X/149/1/012217.

[17] VeeraKrishna/M and G.Subba Reddy Unsteady MHD convective flow of Second grade fluid through a porous medium in a Rotating parallel plate channel with temperature dependent source, IOP Conf. Series: Materials Science and Engineering, vol. 149, p. 012216, 2016. DOI: 10.1088/1757899X/149/1/012216.

[18]. Veera Krishna.M., B.V.Swarnalathamma and J. Prakash, "Heat and mass transfer on unsteady MHD Oscillatory flow of blood through porous arteriole, Applications of Fluid Dynamics, Lecture Notes in
Mechanical Engineering, vol. XXII, pp. 207-224, 2018. Doi: 10.1007/978-981-10-5329-0_14.

[19]. M.Veera Krishna, G.Subba Reddy, A.J.Chamkha, "Hall effects on unsteady MHD oscillatory free convective flow of second grade fluid through porous medium between two vertical plates," Physics of Fluids, vol. 30, 023106 (2018); doi: 10.1063/1.5010863 